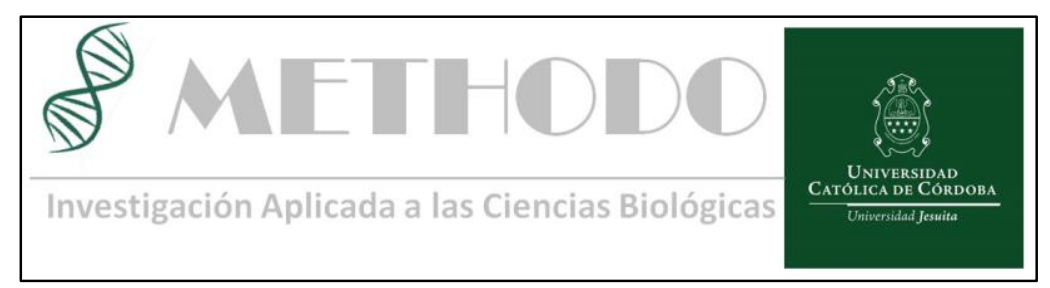

ARTICULO ORIGINAL Methodo 2018;3(1): 12-17

DOI: $10.22529 / \mathrm{me} .2018 .3(1) 04$

Recibido 26 Sep 2017 | Aceptado 05 Mar 2018 | Publicado 30 Mar 2018

\title{
Hernioplastias inguinales transabdominal Preperitoneal: análisis de una serie de casos.
}

\section{Transabdominal inguinal hernioplasties Preperitoneal: analysis of a series of cases.}

\author{
Biurrun Chamale $\mathrm{J}^{1}$, Bruno MA ${ }^{1}$, Olmedo $\mathrm{I}^{1}$, Picón Molina $\mathrm{H}^{1}$, Palencia $\mathrm{R}^{1}$, \\ Doniquian $\mathrm{AM}^{1}$.
}

\begin{abstract}
Resumen:
La hernia inguinal tiene una incidencia entre 6,3 y 23,5 hernias por 10.000 habitantes, siendo uno de los procedimientos más comunes en cirugía, con más de 20 millones de reparaciones globales por año. Una de las técnicas de abordaje por videolaparoscopía es la vía transabdominal preperitoneal (TAPP)

Objetivos:

Describir la experiencia en una serie de casos de cirugía de hernia plastia por la vía TAPP.

Pacientes y método:

Diseño del estudio: observacional descriptivo.

Población: pacientes operados de hernioplastías inguinales por vía laparoscópica en la Clínica Universitaria Reina Fabiola, Córdoba, Argentina, durante el periodo de febrero de 2016 a febrero de 2017.

Resultados:

Se estudiaron 47 pacientes operados; 44 (93.2\%) hombres, con un promedio de edad de 40.53 (18-65) años y de índice de masa corporal de $28.6(24-51.2) \mathrm{kg} / \mathrm{m} 2$. Se operaron 89 hernias, $42(89.3 \%)$ bilaterales y $5(10.7 \%)$ unilaterales. Al $100 \%$ de los pacientes se les practicó la técnica TAPP. Existieron $3(3.33 \%)$ recidivas. No hubo mortalidad en la serie. Conclusiones:

Las hernioplastías inguinales laparoscópica presentan una curva de aprendizaje rápida en cirujanos experimentados, con bajas tasas de morbilidad y recidivas.
\end{abstract}

Palabras claves: hernioplastia, hernioplastia inguinal laparoscópica, preperitoneal, transabdominal.

\footnotetext{
Abstract

The inguinal hernia has an incidence between 6.3 and 23.5 hernias per 10,000 inhabitants, being one of the most common procedures in surgery, with more than 20 million global repairs per year. One of the techniques of videolaparoscopy approach is the transabdominal preperitoneal (TAPP)

Objectives:

To describe the experience in a series of cases of hernia surgery by TAPP.

Patients and method:

Study design: descriptive observational.

Population: patients undergoing laparoscopic inguinal hernioplasty at the Clínica Universitaria Reina Fabiola, Córdoba, Argentina during the period from February 2016 to February 2017.
}

Revista Methodo: Investigación Aplicada a las Ciencias Biológicas. Universidad Católica de Córdoba Jacinto Ríos 571 Bo Gral. Paz. X5004FXS. Córdoba. Argentina. Tel.: (54) 3514517299 / Correo: methodo@ucc.edu.ar / Web: methodo.ucc.edu.ar | ARTICULO ORIGINAL Methodo 2018;3(1): 12-17 
Biurrun Chamale J, Bruno MA, Olmedo I, Picón Molina H, Palencia R, Doniquian AM. Hernioplastias inguinales transabdominal Preperitoneal: análisis de una serie de casos

\section{Results:}

47 operated patients were studied; $44(93.2 \%)$ men, with an average age of 40.53 (18-65) years and a body mass index of $28.6(24-51.2) \mathrm{kg} / \mathrm{m} 2$. We operated 89 hernias, $42(89.3 \%)$ bilateral and $5(10.7 \%)$ unilateral. $100 \%$ of the patients underwent the TAPP technique. There were $3(3.33 \%)$ recurrences. There was no mortality in the series.

\section{Conclusions:}

Laparoscopic inguinal hernioplasty has a rapid learning curve in experienced surgeons, with low morbidity and recurrence rates.

Key words: hernioplasty, laparoscopic Inguinal Hernioplasty, preperitoneal, transabdominal.

1. Servicio de Cirugía General, Clínica Universitaria Reina Fabiola, Universidad Católica de Córdoba, Argentina. Correspondencia: Javier Biurrun Chamale. Clínica Universitaria Reina Fabiola. Oncativo 1248 -X5004FHP- Córdoba, Argentina. e-mail: javierbiurrunchamale@gmail.com

\section{Introducción}

La hernia inguinal presenta una incidencia entre 6,3 y 23,5 casos por 10.000 habitantes, siendo de los procedimientos más comunes en cirugía ${ }^{1}$, con más de 20 millones de reparaciones anuales ${ }^{2}$.

La primera reparación herniaria laparoscópica fue descripta por Ger en $1982^{3}$. Actualmente existen dos técnicas de abordaje operatorio, la primera, descripta por McKernan en 1993, por vía extraperitoneal (TEP) y la segunda por Arregui en 1992 por vía transabdominal preperitoneal (TAPP) $)^{45}$

El objetivo de este estudio es describir la experiencia inicial por la vía TAPP en el Servicio de Cirugía General de la Clínica Universitaria Reina Fabiola, Córdoba, Argentina en las primeras 89 hernias operadas de manera consecutiva.

\section{Material y método}

Diseño Estudio observacional, descriptivo.

Pacientes:

Pacientes intervenidos mediante hernioplastías inguinales por vía laparoscópica en la Clínica Universitaria Reina Fabiola, entre febrero de 2016 y mayo de 2017.

Se incluyeron todos los pacientes con hernias inguinales sintomáticas, primarias, con una edad mayor de 16 años. Se excluyeron hernias inguinales recidivadas, inguinoescrotales, incarceradas, pacientes con múltiples antecedentes quirúrgicos en abdomen inferior, pacientes con elevado riesgo anestésico, según escala de la Asociación Americana de Anestesiología (A.S.A.), y quienes no aceptaron

participar en el estudio mediante consentimiento informado.

\section{Método:}

Todos los procedimientos fueron realizados por el mismo cirujano, el cual cuenta con experiencia de más de 1500 procedimientos laparoscópicos más un cirujano en formación.

Los pacientes fueron internados el mismo día de la cirugía. Se realizó antibioticoprofilaxis con cefazolina (1 gr. intravenoso 30-45 min antes de la cirugía). No se realizó profilaxis antitrombótica ni rasurado del sitio operatorio. No se utilizó sonda vesical en ningún caso, se estimuló a la micción preoperatoria inmediata.

Técnica quirúrgica

Los pacientes fueron colocados en decúbito dorsal, con inclinación cefálica de $20^{\circ}$ de la mesa operatoria. El cirujano y su ayudante se ubicaron en forma contra lateral a la hernia a operar.

El procedimiento se realizó bajo anestesia general intravenosa con intubación orotraqueal. Todos los pacientes fueron operados con idéntica técnica de abordaje: Transabdominal preperitoneal (TAPP).

Se practicó neumoperitoneo en región supraumbilical por punción con aguja de Veress. Se utilizaron un trocar de $10 \mathrm{~mm}$ para la endocámara y dos trocares de $5 \mathrm{~mm}$ para trabajo en localización paramediana a altura del ombligo.

Para la disección se utilizó energía monopolar, se procedió a apertura peritoneal de lateral a medial, empezando a la altura de la espina ilíaca anterosuperior, a unos $3 \mathrm{~cm}$ por encima de la hernia y llegando al ligamento umbilical medial, el cual fue respetado. Como sitios de reparo anatómicos iniciales se tuvieron en cuenta los vasos epigástricos inferiores, el pubis y el ligamento de Cooper.

La disección del espacio preperitoneal fue amplia, y se obtuvieron los siguientes márgenes: 
Biurrun Chamale J, Bruno MA, Olmedo I, Picón Molina H, Palencia R, Doniquian AM. Hernioplastias inguinales transabdominal Preperitoneal: análisis de una serie de casos

Lateral: $2 \mathrm{~cm}$ de la espina ilíaca antero superior

Medial: espina del pubis

Craneal: $7 \mathrm{~cm}$ por encima del pubis

Caudal: hasta agujero del obturador

Este espacio se dejó libre de tejido graso o adherencias que impidiesen el correcto posicionamiento de la malla. Al identificar los tumores lipomatosos, estos fueron disecados y reducidos. Los sacos herniarios directos grandes fueron evaginados. Se redujeron la totalidad de los sacos herniarios indirectos.

Se colocaron mallas protésicas de poliéster macroporosas (Parietex $\AA$, Covidien, Chicago, EE. UU.) o de polipropileno de mediana densidad de $15 \times 15 \mathrm{~cm}$, (Ultrapro®, Johnson y Johnson, EE.UU).

Para la fijación se emplearon, grapas absorbibles (ETHICON SECURETRAP®, Johnson y Johnson,EE. UU.). Esta se llevó a cabo en el ligamento de Cooper y en la parte superior de medial a lateral.

El peritoneo fué cerrado con grapas en la mayoría de los pacientes y a los restantes se le realizó surget de poliglactina 910 3-0.

$\mathrm{Al}$ finalizar la cirugía se infiltraron los puertos de acceso laparoscópico con clorhidrato de bupivacaína al $0.5 \%$.

Seguimiento

El postoperatorio se cursó por ambulatorio. Se prescribió analgesia con Ketoroloac (20 mg cada 8 h por 3 días) y se llevó a cabo un registro de necesidad de analgesia adicional.

Los pacientes fueron valorados por el cirujano a los 10, 30, 180 y 365 días del procedimiento. Al momento del estudio, todos los pacientes presentaban al menos seguimiento de 6 meses.

Variables

Las variables estudiadas fueron:

Edad

Sexo

Índice de masa corporal (IMC)

Tipo de hernia (unilateral, bilateral)

Tiempo operatorio

Complicaciones (según clasificación de

Clavien-Dindo modificadas en 2009)

Dolor postoperatorio (escala visual análoga-EVA

Analgesia de rescate

Retorno a la actividad habitual

Recidiva

Se prestó especial interés al tiempo quirúrgico, dolor, requerimiento de analgesia adicional y reincorporación a las actividades habituales.

Se definió como complicación a toda desviación del curso postoperatorio ideal, no inherente al procedimiento y no debida a una falla terapéutica presentado en un periodo de 365 días desde la cirugía. Se consideraron complicaciones tempranas, las que se suscitaron en los primeros 30 días del procedimiento y a complicaciones tardías a las comprendidas a partir de este período.

Se consideró como hernia recidiva a toda recurrencia de tumor inguinal que aumentan de tamaño con maniobras de Valsalva, sea este asociado o no a dolor. El diagnóstico se realizó con Tomografía Computada. Las hernias fueron clasificadas en: directas, indirectas, mixtas.

La información se recopiló en base de datos prospectivos.

\section{Análisis estadístico}

El análisis estadístico fue realizado utilizando el programa SPSS, los resultados de variables cuantitativas se expresaron como medias y rangos. Las variables cualitativas se expresaron como porcentajes.

\section{Resultados}

Durante el período de estudio se operaron de forma consecutiva y electiva 47 pacientes; 44 (93.2\%) fueron hombres. La media de edad fué 40.53 (rango de 18-65) años. La media de IMC fue de $28.6(24-51.2) \mathrm{kg} / \mathrm{m} 2$.

Se operaron un total de 89 hernias. $42(89.3 \%)$ fueron bilaterales y $5(10.7 \%)$ unilaterales. Al $100 \%$ de los pacientes se les practicó la técnica TAPP. No se registraron conversiones de reparaciones laparoscópicas a convencional.

Se repararon $44(49 \%)$ hernias indirectas, 18 $(21 \%)$ directas y $27(30 \%)$ mixtas.

El tiempo quirúrgico promedio en las hernioplastías unilaterales fué de 39 (20-70) minutos y en las bilaterales de 78 (35-120) minutos.

Se utilizaron mallas de polipropileno en 82 $(93.2 \%)$ hernioplastías y de polyester y colágeno absorbible en $7(6.8 \%)$.

En el $100 \%$ de las hernioplastías se utilizaron grapas absorbibles de fijación.

En la serie se presentaron $8 \quad(17 \%)$ complicaciones. $6(75 \%)$ fueron tempranas (tabla 1). Ninguno requirió prolongar el periodo de internación. Existieron 2 (25\%) complicaciones tardías (tabla 1). Ninguno de estos requirió internación, siendo manejados de forma ambulatoria con tratamiento médico.

En la serie se registró una necesidad de analgesia adicional, en concepto de aumento de dosis o prolongación en días de tratamiento, en 10 (23\%) pacientes.

El promedio de días para retornar a sus actividades habituales fue de 10.7 (7-21) días. El atraso en la reincorporación se relacionó con un requerimiento de analgesia adicional.

Se realizó un seguimiento postoperatorio del 100 $\%$ de los pacientes. 
Biurrun Chamale J, Bruno MA, Olmedo I, Picón Molina H, Palencia R, Doniquian AM. Hernioplastias inguinales transabdominal Preperitoneal: análisis de una serie de casos

En el estudio se presentaron un total de $3(3.33 \%)$ recidivas en el seguimiento. El promedio de tiempo de diagnóstico de la recidiva fue de 5 meses.

La presente serie no se presentó mortalidad.

\section{Discusión}

En nuestra serie hubo una predominancia de pacientes de sexo masculino, entre la tercera y cuarta décadas de la vida, lo cual está de acuerdo con lo reportado en la literatura mundial ${ }^{6}$.

El mayor número, corresponde a hernias bilaterales, debido a que las aseguradoras de salud solo autorizan las reparaciones laparoscópicas cuando la patología es bilateral, a pesar que las guías de práctica médica ${ }^{6}$ actuales estimulan las reparaciones laparoscópicas en todo defecto inguinal.

Se presentó un mayor índice de defectos indirectos en coincidencia con lo publicado por Simons ${ }^{6}$.

Nuestro grupo de trabajo optó por iniciar la serie de reparaciones laparoscópicas con técnica TAPP, debido a que esta insume menos costo al no requerir el uso de balón de disección y al necesitar un menor entrenamiento en la técnica, conforme a lo sugerido en la literatura por McCormack $^{7}$ y Misra ${ }^{8}$.

La serie presentó un número decreciente de complicaciones conforme aumentaba la experiencia en la técnica y el incremento la curva de aprendizaje. Lo que es coincidente con lo publicado en los reportes mundiales ${ }^{6}$.

Vale mencionar que la literatura no es precisa en cuanto al número exacto de procedimientos que es necesario para considerar haber alcanzado la curva de aprendizaje, así lo manifiesta Simons ${ }^{6}$ en su publicación. Por otro lado, Voitk ${ }^{9}$ y Barrat $^{10}$ sugieren que el número necesario para alcanzarla serían al menos 50 hernioplastías laparoscópicas para disminuir considerablemente las complicaciones intra $\mathrm{y}$ postoperatorias y el tiempo quirúrgico.

Acorde a la literatura global, el mayor porcentaje de complicaciones, se presentó a corto plazo, es decir dentro del primer mes de cirugía, tal y como se encuentra en la literatura ${ }^{11}$, coincidente con lo observado en nuestra serie.

El dolor postoperatorio retrasaría la reincorporación a actividades cotidianas, según pudimos observar en nuestro estudio, hallazgo que es coincidente con la literatura actual según Poobalan ${ }^{12}$, Roll ${ }^{13}$ y Belyansky ${ }^{14}$ quienes mencionaron que la fijación de las mallas con un mayor número de grapas espiroideas absorbibles serían los responsables del incremento del dolor postoperatorio. En la actualidad hay múltiples estudios que comparan el empleo de grapas espiroideas frente a la fijación con pegamentos biológicos y sintéticos, lo que revela resultados a favor de la fijación no invasiva, al ofrecer menor dolor posoperatorio con índices de recidivas similares $6,12,15,16$

Contrariamente a lo antes mencionado, Zhang ${ }^{17}$ y Bresnahan $^{18}$ demostraron una disminución significativa en el tiempo operatorio, no así en el dolor en la colocación de prótesis con fijación no invasiva.

En nuestros pacientes, la reincorporación a las actividades habituales se llevó a cabo, en promedio, a los 10.7 días, a diferencia de lo publicado por Callesen ${ }^{19}$ y Salcedo ${ }^{20}$ quien tuvo una media de reincorporación de 14/21 y de 14.9 días respectivamente.

Presentamos un porcentaje de recidiva de 3.33 $\%$, cercano con lo reportado por Wright $^{21}$ y Maddern $^{11}$ quienes presentaron un índice de $1.5 \%$, una vez superada la curva de aprendizaje. En la bibliografía se atribuyen como principales causas de recidivas al uso de mallas pequeñas, mal cortadas o de material inadecuado, mal sistema de fijación, tamaño de presentación de la hernia ${ }^{6,8,9}$.

Las limitaciones en este estudio son la naturaleza retrospectiva, el carácter descriptivo del diseño y el número reducido de pacientes, aunque aporta información valiosa en este campo, debido a que la mayoría de las publicaciones en esta área son en la actualidad series con acotado número de pacientes y en estadio tempranas de experiencia.

\section{Conclusión}

Las hernioplastías inguinales por vía laparoscópica presentan una curva de aprendizaje corta en cirujanos experimentados, con bajas tasas de morbilidad y recidivas. Lo que convierte este procedimiento en el estándar de oro en el manejo de defectos parietales inguinales. 
Biurrun Chamale J, Bruno MA, Olmedo I, Picón Molina H, Palencia R, Doniquian AM. Hernioplastias inguinales transabdominal Preperitoneal: análisis de una serie de casos

\section{Bibliografía}

1. Bernhardt $\mathrm{G}$, Gruber $\mathrm{G}$, Molderings B, Cerwenka H,Glehr M, Giessauf C, et al. Healthrelated quality of life ater TAPP repair for the sportsmen's groin. Surg Endosc (2014) 28:439-446

2. Kulacoglu H. Current options in inguinal hernia repair in adult patients. Hippokratia 2011; 15: 223-31.

3. Ger R. The management of certain abdominal herniae by intra-abdominal closure of the neck of the sac. Preliminary communication. Ann R Coll Surg Engl. 1982;64:342-4.

4. McKernan JB, Laws HL. Laparoscopic repair of inguinal hernias using a totally extraperitoneal prosthetic approach. Surg Endosc 1993; 7: 26-28.

5. Arregui ME, Davis CJ, Yucel O, Nagan RF. Laparoscopic mesh repair of inguinal hernia using a preperitoneal approach: a preliminary report. Surg Laparosc Endosc. 1992;2:53-8.

6. Simons MP, Aufenacker T, BayNielsen M, Bouillot JL,Campanelli G, Conze J, et al. European Hernia Societyguidelines on the treatment of inguinal hernia in adultpatients. Hernia. 2009;13:343-403.

7. McCormack K, Wake BL, Perez J, Fraser C, Cook J, McIntosh E,et al. Laparoscopic surgery for inguinal hernia repair:Systemic review of effective- ness and economic evaluation. Health Technol Assess. 2005;9:1-203.

8. Misra MC, Kumar S, Bansal VK. Total extraperitoneal (TEP)mesh repair of inguinal hernia in the developing world:Comparison of low-cost indigenous balloon dissection versusdirect telescopic dissection: A prospective randomizedcontrolled study. Surg Endosc. 2008;22:1947-58.

9. Voitk AJ. The learning curve in laparoscopic inguinal herniarepair for the community general surgeon. Can J

Surg. 1998;41:446-50.

10. Barrat C, Voreux JP, Occelli G, Catheline JM, Champault G.Effects of surgical education and training on the results oflaparoscopic treatment of inguinal hernias. Chirurgie.1999;124:298-303.

11. Maddern GJ, Rudkin G, Bessell JR, Devitt P, Ponte L.

Acomparison of laparoscopic and open hernia repair as a daysurgical procedure. Surg Endosc. 1994;8:1404-8.

12. Poobalan AS, Bruce J, Smith WC, King PM, Krukowski $\mathrm{ZH}$,Chambers WA. A review of chronic pain after inguinalherniorrhaphy. Clin J Pain. 2003;19:48-54.

13. Roll S. A global vision for hernia repair improvement. GenSurg News. 2012;39:01.

14. Belyansky I, Tsirline V, Klima D, Walters A, Lincourt A,Heniford T. Prospective, comparative study of postoperativequality of life in TEP, TAPP, and modified Lichtenstein repairs.Ann Surg. 2011;254:709-15.

15. Lau H. Fibrin sealant versus mechanical stapling for meshfixation during endoscopic extraperitoneal inguinalhernioplasty: A randomized prospective trial. Ann Surg.2005;242:670-5.

16. Kukleta JF, Freytag C, Weber M Efficiency and safety of meshfixation in laparoscopic inguinal hernia repair using $\mathrm{n}$ butylcyanoacrylate: Long-term biocompatibility in over 1,300 meshfixations. Hernia. $2012 ; 16: 153-62$

17. Zhang $\mathrm{C}$, Li F, Zhang H, Zhong W, Shi D, Zhao Y. Selfgrippingversus sutured mesh for inguinal hernia repair: A systematicreview and metaanalysis of current literature. $\mathrm{J}$ Surg Res.2013;185:653-60 
Biurrun Chamale J, Bruno MA, Olmedo I, Picón Molina H, Palencia R, Doniquian AM. Hernioplastias inguinales transabdominal Preperitoneal: análisis de una serie de casos

18. Bresnahan E, Bates A, Wu A Reiner M, Jacob B. The use ofself-gripping (ProgripTM) mesh during laparoscopic totalextraperitoneal (TEP) inguinal hernia repair: A prospectivefeasibility and longterm outcomes study. Surg Endosc.2015;29:2690-6. Epub 2014 Dec 18. Erratum in: Surg Endosc.2015 Sep;29(9):2697.

19. Callesen T. Inguinal hernia repair: Anaesthesia, pain andconvalescence. Dan Med Bull. 2003;50:203-18.
20. Salcedo-Wasicek MC, Thirlby RC. Postoperative course afteringuinal herniorrhaphy. A case-controlled comparison ofpatients receiving workers compensation vs. patients withcommercial insurance. Arch Surg. 1995;130:29-32.

21. Wright D, Paterson C, Scott N, Hair A, O'Dwyer PJ. Fiveyearfollow-up of patients undergoing laparoscopic or open groinhernia repair: A randomized controlled trial. Ann Surg.2002;235:333-7. 